\title{
A Low Cost and Easy to Use Setup for Foot Scanning
}

\author{
C. Lovato*, E. Bissolo, N. Lanza, A. Stella, A. Giachetti \\ Dep. of Computer Science, University of Verona, Verona, Italy
}

http://dx.doi.org/10.15221/14.365

\begin{abstract}
We present a low cost acquisition setup for the accurate reconstruction of feet and shoe lasts geometry. It is based on the use of a single close range depth sensor (Primesense Carmine 1.09) that is moved around the object using a PC-controlled mobile robot.

The mobile robot is built using a simple electric car constrained to move in a circular guide and controlled by an Arduino Uno board allowing the custom software to control the engine's speed.

In the acquisition phase multiple point clouds are captured and registered in a common reference frame. The vehicle trajectory has been designed to maximize the reconstruction accuracy and robustness against changes in the scene illumination.

Registered point clouds are filtered in order to remove the floor, reduce the noise and refine the alignment, and are finally merged in an unique simplified cloud that can be triangulated with standard techniques. The resulting foot model is then aligned in a standard way and segmented identifying relevant landmarks for measurement.
\end{abstract}

Keywords: Foot scanning, depth sensors, registration, shoe lasts.

\section{Introduction}

One of the recent trends in the context of the garment and shoe industry is definitely the use of digital tools to reconstruct $3 \mathrm{D}$ models of the specific subject from which accurate measurements can be obtained. Through these measures, it is possible to find the best shapes and sizes of shoes for a determined population based on specific statistics of the real anatomical variations of the subjects.

Specific measurement system for geometric foot parameters have been realized and used for population studies and industrial applications $[1,2]$

In addition, the acquisition, analysis and visualization of 3D models can be of great impact in ecommerce, allowing the creation of virtual trials/fittings on clothes and shoes. While in a recent past the high cost of the 3D acquisition devices limited the diffusion of digital anthropometry tools, the advent of new and cheap depth sensors and dense stereo software packages are making digital 3D reconstruction widely available for a variety of applications.

A cheap and easy to use scanner for the acquisition of foot shape can be extremely useful for shoe manufacturers, in order to enable customers to capture accurately their feet shape for custom shoe making or e-commerce applications. Classical foot scanners are, however, expensive and difficult to place in many shops or homes.

Several methods have been therefore recently proposed in order to obtain cheap and sufficiently accurate foot scanners. In [3] a remote service for 3D reconstruction from uploaded video has been proposed. In [4] a foot scanner based on Kinect sensor has been proposed.

Many free or low cost software packages can also be used to turn low cost depth sensors into 3D scanners, but manual acquisition of feet with a portable devices is not easy and the online reconstruction of the geometry proposed in some applications like the KinectFusion [5] may result in inaccurate geometries when capturing a complete object due to registration error accumulation.

In this paper we propose a scanning solution based on a cheap depth sensor that is able to recover easily and quickly 3D models of human feet and shoe lasts with accuracy sufficient to perform the measurements required for shoe last design and shoe sizing, thanks to an accurate offline registration of captured 3D views and an automated acquisition of the multiple views.

The design of the system has been realized in a collaborative project including the Departments of Computer Science and the Department of Neurological and Movement Sciences of the University of Verona and a consortium of local shoe manufacturers. We plan to realize not only 3D reconstruction tools directly exploitable by the companies to sell shoes or to design custom lasts, but also to investigate the relationship between the shape of the foot and the shape of the lasts used shoes comfortably fitting the foot shape.

*email: chr_lovato@yahoo.it, andrea.giachetti@univr.it 


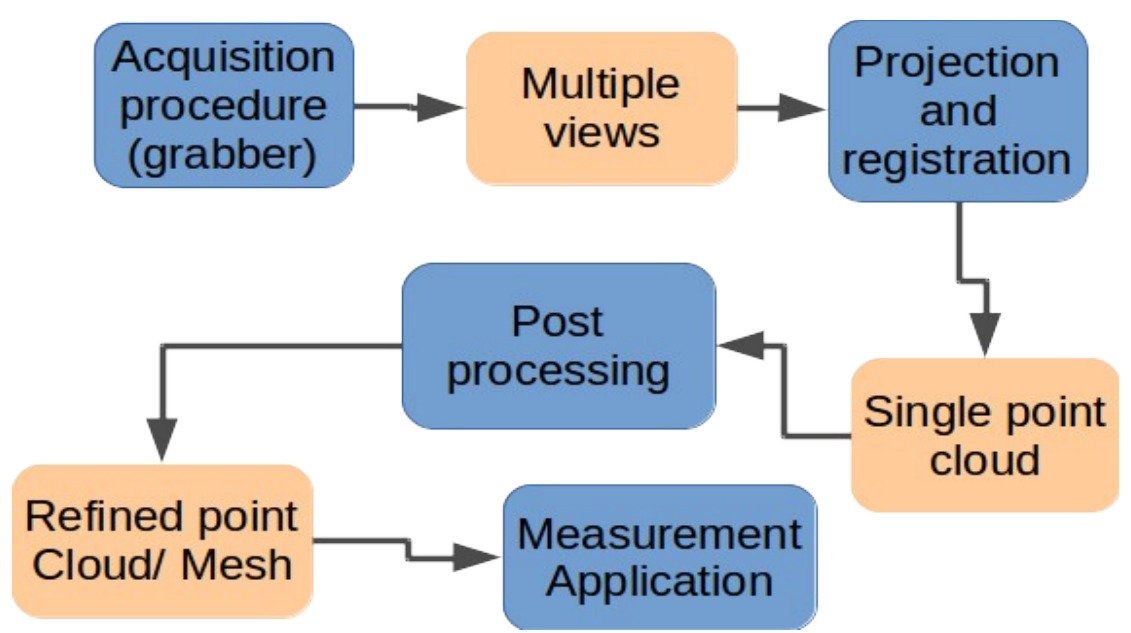

Figure 1: Block diagram of the acquisition pipeline

\section{System design}

The basic idea driving the of our prototype was to realize a cheap and easy to use system for a quick and fully automatic reconstruction. To guarantee low cost and limited user interaction we decided to use a single 3D depth sensor making it automatically rotating around the object to be scanned.

The depth sensor acquires, moving along its planned trajectory, several views of the object that are finally registered, cleaned and merged in order to obtain the 3D reconstruction of the complete model. Additional software components perform then the visualization and the desired measurements on the $3 \mathrm{D}$ shape and is different for the processing of foot, shoe last or shoe models. The diagram of the processing pipeline is represented in Figure 1.

In the next sections we will describe the hardware and software components used or realized according to the scheme of Figure 1.

\section{Hardware components}

\section{D Acquisition Device}

The depth sensor employed in our prototype is currently a PrimeSense Carmine 1.09. This sensor was preferred to the Microsoft Kinect for its features of compactness, lightness and reduced power consumption, which allow to feed it with the simple USB socket, instead of requiring a separate power as in the case of Kinect devices. Its short range sensing feature ensure the possibility of building a compact system with sufficient resolution for our application (about $1 \mathrm{~mm}$ ). As the technology of depth sensor is quickly evolving it is clearly possible that in the near future other new low cost depth sensing device with improved quality will be available in the market, and it is worth noting that the replacement of the sensor in our system should not create particular problems if sensor size is small and drivers are available.

\section{Scanner platform and moving vehicle}

The geometric characteristics of the platform are primarily derived from the sensor optical features, which has a nominal range of $35 \div 140 \mathrm{~cm}$, the size of the object to be scanned and the accuracy requirements. Analyzing the geometry of the setup we estimated that a good acquisition of our models can be obtained when the sensor is maintained at a distance of about $80 \mathrm{~cm}$ from the rotation center, at height between 40 and $80 \mathrm{~cm}$. Lower heights results in poor acquisitions due to too small angles between optical axis of the sensor and surface normals. Given these we realized a lightweight and easily mountable wooden lane that support the carriage with the depth sensor in its path around the model. The path covers an angle of 270 degrees along a circumference with a radius of $80 \mathrm{~cm}$.

The vehicle moving on this path is derived by a small toy battery powered electric car modified adding platforms to host sensor and control devices and custom wheels adapted to the platform. The car is provided with two additional wheels on the external side of the curve that drive the vehicle along the 


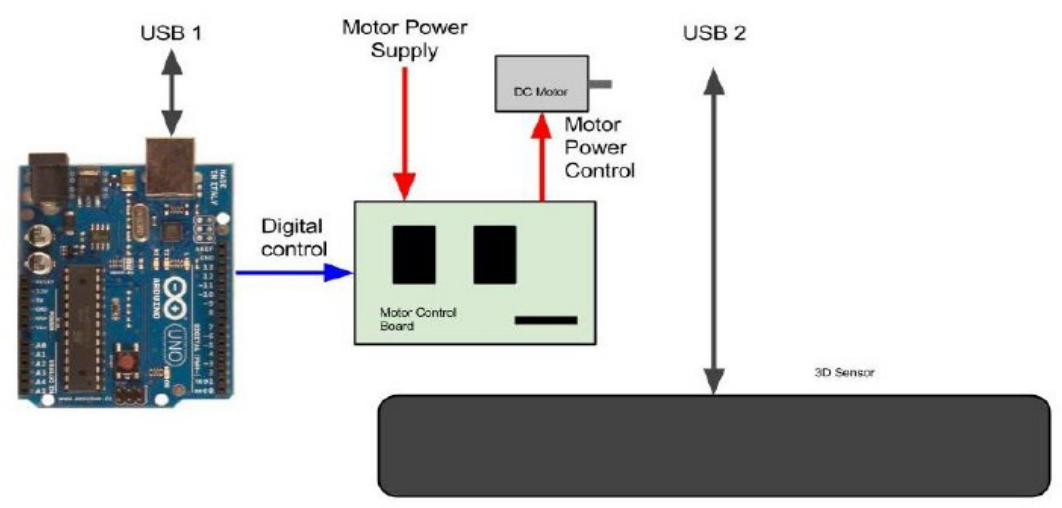

Figure 2: Scheme of the control system

circular path. Inside the acquisition circle a simple platform with printed landmarks (24 squared patterns in the current version) is placed in order to facilitate the alignment of multiple views. Landmarks have been obtained using the ArUco Augmented Reality toolkit [6].

\section{Vehicle control hardware}

The electronic components used to control the vehicle from the PC user interface are also simple and cheap. They consist of an Arduino Board Uno equipped with Motor Shield and a custom Motor control board. The motor control board, which is necessary to provide adequate electrical power to the motor, has been realized with few electronic components. The tabs and the sensor were arranged as shown in Figure 1, where it is also shown the diagram of connections.

The Arduino board controls the motors according to the commands that are provided by the interface that runs on the PC (see Section 3).

The scheme of the scanner vehicle is shown in Figure 2. Figure 3 shows the physical configuration of the vehicle in action and the mounted acquisition platform with the wooden lane and the platform with the ArUco generated markers.

\section{3D acquisition software}

The design of the software tool for controlling the shape capture is modular so that each component of the system could be easily changed without affecting the whole application. So we have an user interface controlling the acquisition and synchronizing vehicle movement and depth image capture and separate modules to reconstruct the geometry from the captured depth maps registering multiple views and processing the point clouds. Another interesting aspect of the software developed is that all the components have been realized using cross-platform toolkit so that the system can run indifferently on Windows, Mac or Linux machines.

\section{Control user interface}

The user interface has been realized using the Processing2 environment [7], which allowed us to program the Arduino and capture images (via the library Simple-OpenNI [8]) in a synchronized way using a single interface specification.

Role of the control interface is to capture the multiple views required for the full shape reconstruction, that for 3D sensors like those used by us consist of a depth image coupled with a corresponding RGB image. In the current implementation we capture both the images with a resolution of $640 \times 480$ pixels. As the view acquisitions are synchronized with the movement of the carriage, it is important, in order to properly design the acquisition timing, to set the resolution (that changes the sensor acquisition rate) and the vehicle speed to reasonable values given the time and accuracy constraints of the application. An example of depth-RGB images acquired is visible in Figure 4.

Through the interface it is possible to start/stop the scanning sequence and to bring the device to the starting position. Different protocols relative modifying vehicle speed and number of captured frames can be easily implemented and are being tested in order to achieve better resolution/accuracy or faster acquisition speed. 


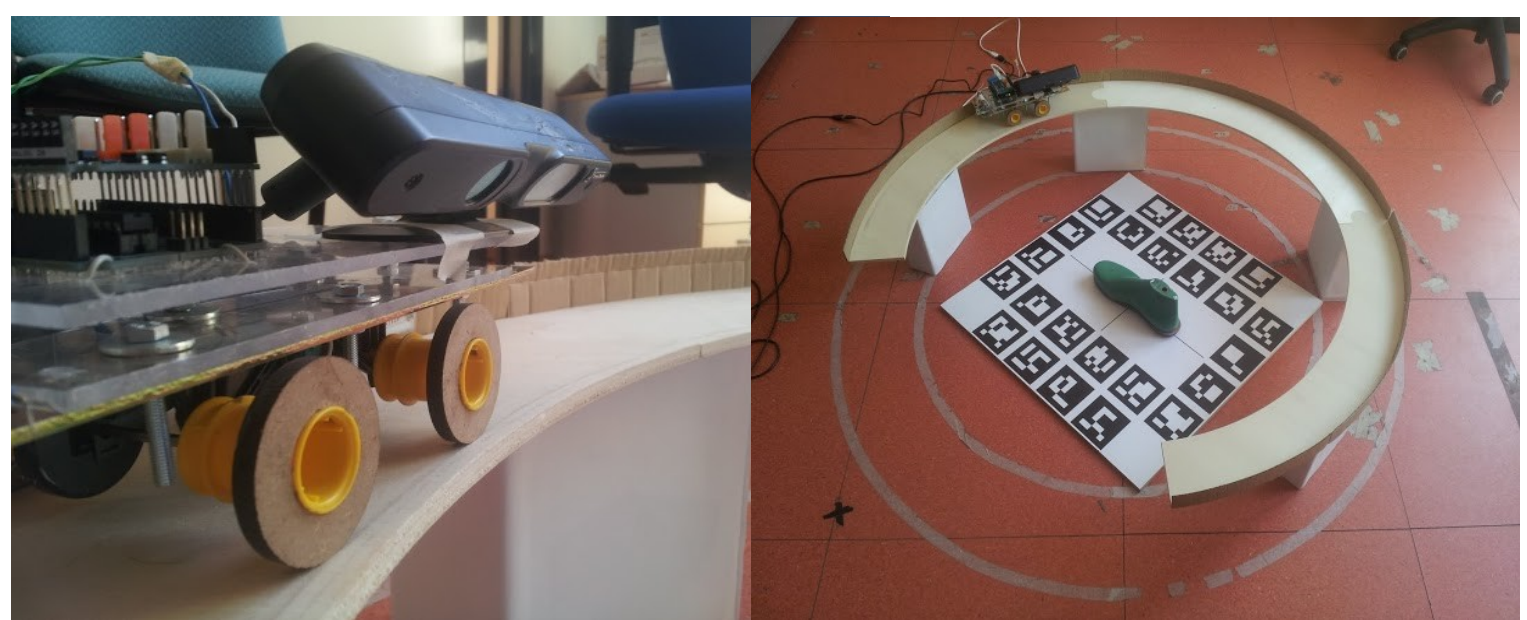

Figure 3: Left: the moving vehicle setup. Right the scanner prototype with the platform with markers

\section{Depth image processing and 3D reconstruction}

Depth images are then processed by a separate module registering different views in a reference system and building the 3D model. This software module is based on the Point Cloud Library (PCL) [9], which provide a wide variety of methods to process and display images and point clouds captured by 3D sensors.

First depth images are converted in point clouds each one placed in its own reference system using intrinsic parameters of the capturing devices. In the current implementation we use the default calibration, but we plan to replace this one with a specific calibration taking into account also radial distortion in order to increase the resulting accuracy.

Resulting point clouds are then registered in the reference system of the platform thanks to the use of markers recognized in the platform. For the generation of the pattern with the markers and the subsequent recognition of the markers on the images the augmented reality toolkit ArUco [6], that allows the user to create tables containing multiple markers, with known 3D corner coordinates. Using the library functions it is then possible to obtain from RGB images the corner position with subpixel resolution, so that we can put them in correspondence with the depth values and with the points of the clouds. Using PCL methods point clouds can be then easily put in an unique reference system centered in the middle of the board, on which the object to be acquired should have been positioned. The alignment is then refined using the Iterative Closest Point (ICP) algorithm. An example of markerbased registration of eight views creating a complete 3D model is shown in Figure 5.

We also developed also a different reconstruction module allowing an accurate point cloud registration without the use of the markers. It exploits the knowledge of the acquisition sequence so that we can assume that two point clouds acquired at consecutive frames are sufficiently close and can be aligned in a single reference system using ICP algorithm. To avoid error accumulation, a hierarchical ICP alignment has been designed so that after a first alignment of couple of consecutive frames, in the following step clouds including points from consecutive frames are registered further

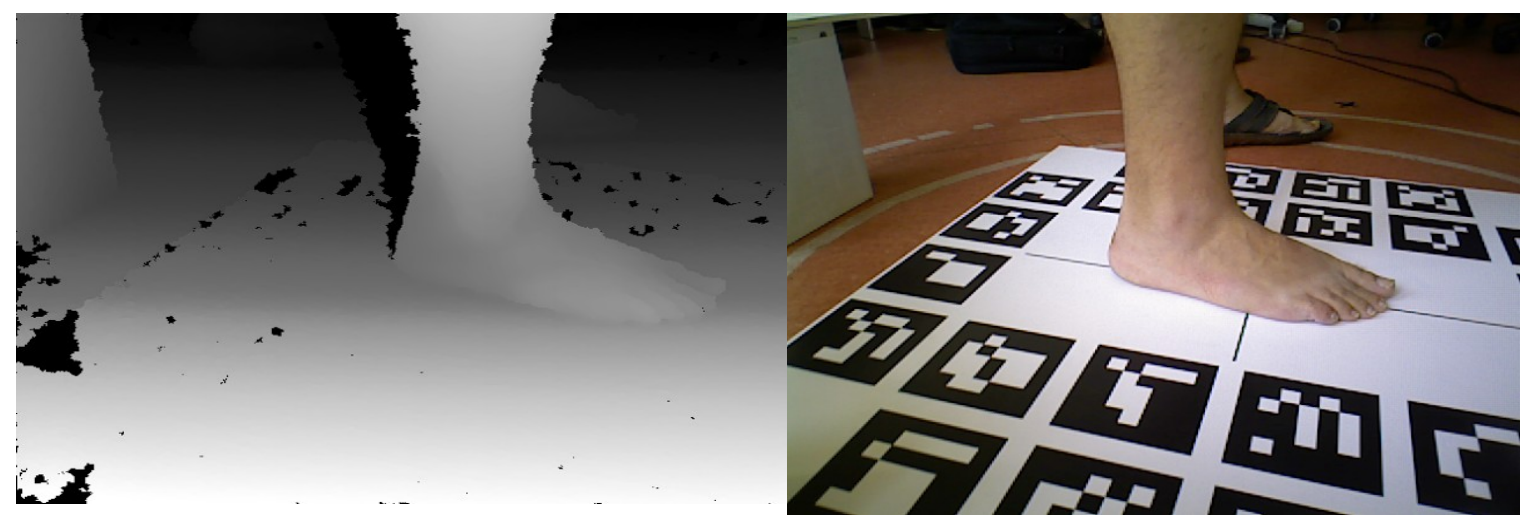

Figure 4: Example depth map (left) with coupled RGB data (right) captured during a foot scanning procedure 


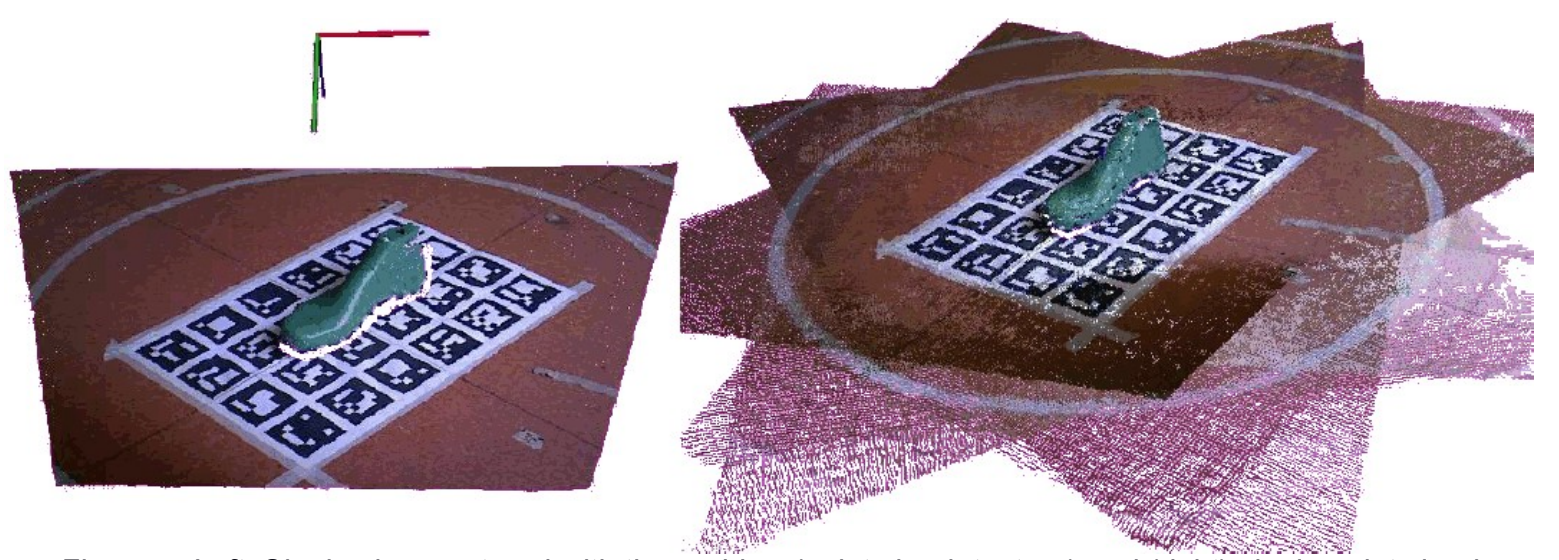

Figure 5: Left: Single view captured with the grabber (point cloud+texture) and (right) single point cloud obtained from multiple view registration (8 in this case)

and the procedure is iterated until an unique point cloud is obtained. This procedure is able to create accurate reconstruction provided that a sufficient number of frames is captured (at least 32 views). When the pattern with markers is available, however, as it can be in standard scanning procedures, we can obtain reconstructions more quickly and accurately with a reduced number of frames (8 frames can be enough).

\section{Point Clouds' post-processing}

Starting from the point cloud resulting from the recording of the different views, the first step is to separate the floor plane from the object and from any spurious signals. This step is performed using standard PCL functions if the alignment is realized without the markets, otherwise it is directly provided by the registration as in the platform reference system the floor plane is simply $z=0$.

In the case in which the subject acquired both a foot or a shoe worn, it is necessary a further step of elimination of the points above a height equal to $15 \mathrm{~cm}$, which leaves unchanged the structures of the malleoli, which can be used subsequently to perform the measurements.

Once the object of interest has been extracted, the PCL Radius Outlier Removal Filter is applied to further clean up the cloud, and subsequently the PCL Moving Least Square Filter that evens out the surface of the object.

The model can be then processed using standard meshing algorithms and evaluated with measurement software. We plan to realize a custom foot measurement system in the near future according to the requirements of shoe manufacturers collaborating with our research group and specific applications for shoe last analysis, design and printing working on processed 3D captured models.

In this preliminary work we only realized a rough measurement interface in order to directly measure and compare some simple parameters for a first estimation of the scanning quality.

This simple measurement module is directly working on the acquired point cloud and performs the following procedures: the first step is to position exactly the model in the space of measurement, by applying a translation such as to bring the heel aligned with the origin of the space. Subsequently the
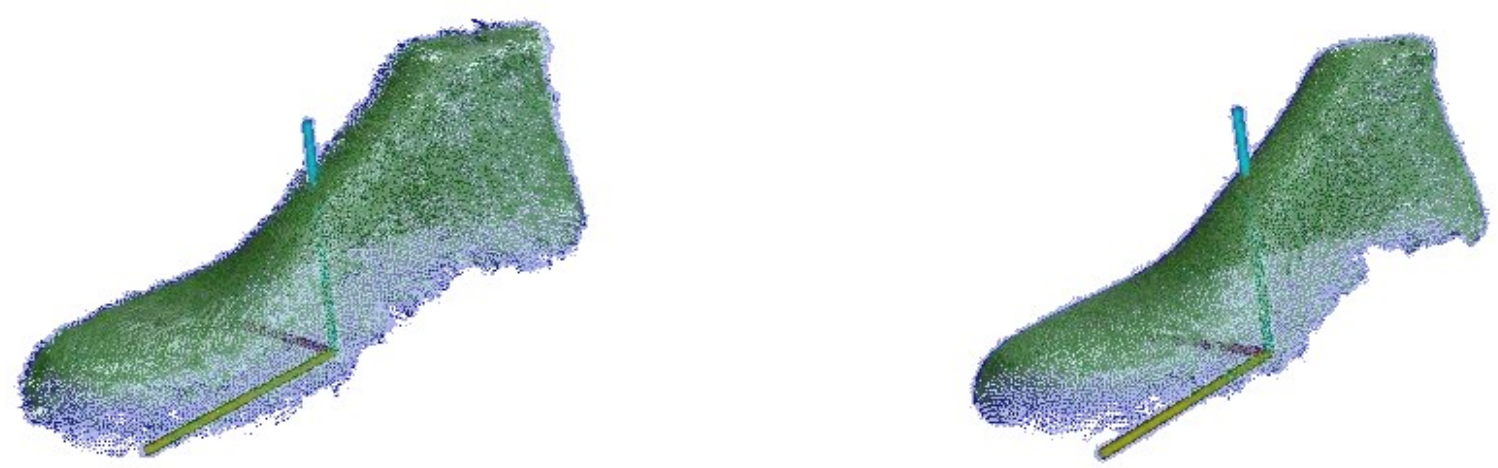

Figure 5: Left: raw point cloud with floor removed and (right) cleaned point cloud 


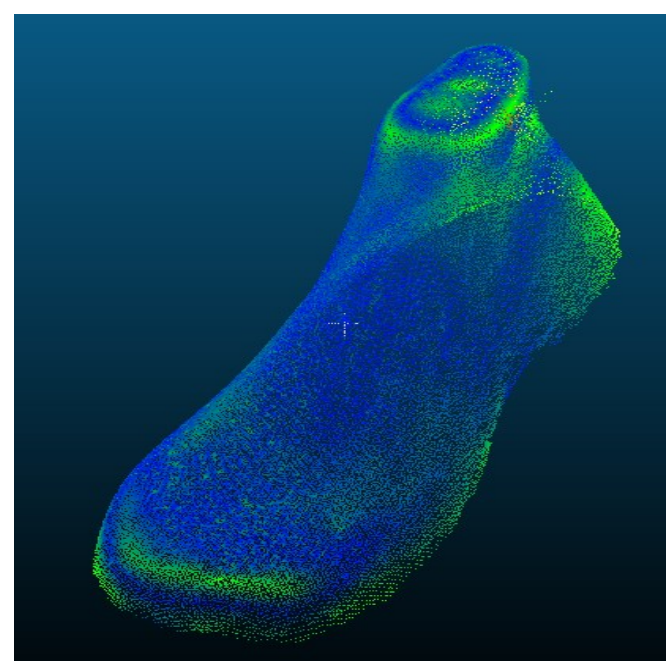

Figure 6: Color coded distance between models of the same shoe last acquired with a Breuckmann BodyScan and our low cost system.

software extrapolates the location of a few anatomical points, as, for example, the extreme points of the front and rear, and the medial and lateral points.

From the annotated landmarks, we can classify left and right feet and perform a few basic measurements such as lengths, widths and heights. Measurements can be saved in CSV format for easy further processing.

We plan to realize in the near future more specific landmark detection and measurement system specialized for industrial needs and anthropometric research.

\section{Evaluation of measurement accuracy}

In order to evaluate if the accuracy of the acquisitions obtained with our system is reasonable for the applications proposed (e.g. shoe sizing, shoe last development and analysis, etc.) we performed some preliminary tests using the 3D analysis tool CloudCompare [10].

First we compared some measurements between landmarks annotated on a shoe last that has been then acquired with the proposed prototype and high resolution structured light scanner device (Breuckmann BodyScan) available of the Department of Neurological and Movement Sciences of the University of Verona. The compared distances were different of less than $2 \mathrm{~mm}$, a value that is already good for an application in the shoe industry, even if the accuracy of the comparison is not optimal due to the necessity of manually picking landmarks in order to extract measurements.

On the same shoe last model we also evaluated the average point distance with the tool included in CloudCompare. The two acquired model were registered with manual initialization (point based) and ICP refinement using the CloudCompare options and then the accurate point distance measurement was performed. The average distances between points was evaluated equal to $1.33 \mathrm{~mm}$ with standard deviation $0.94 \mathrm{~mm}$. A color coded map of the computed distances is represented in Figure 6, showing that errors are mainly located near the upper and lower borders.

The acquisition of the lower part of the feet is clearly a problem for any 3D foot scanning device and clearly also the accuracy at the lower extrema of acquired partial model is a relevant issue. We plan to develop a model based solution for recovering complete models and/or a separate protocol to acquire and register the sole of the foot.

The preliminary values are, however, extremely encouraging, especially considering that the values include also registration errors and a few non removed outlier points, the current software implementation does not implement an accurate device calibration, and a limited number of views has been used for the reconstruction of the model (8).

We plan to refine the software reconstruction software mainly through a new calibration of the sensor device correcting the radial distortion as proposed in [11] and a refinement of the registration procedure. After this step we will perform an accurate validation of the feet and shoe lasts models accuracy. 


\section{Discussion}

The availability of easy to use accurate and cheap scanning system is a fundamental requirement to develop custom applications for shoe manufacturers (e.g. last design for custom shoe making, easy shoe sizing for e-commerce, etc.) and to perform large scale studies on human feet anthropometric variability.

Being interested in both these applications as collaborating with a University department active in human body measurement and with local shoe manufacturers, we developed a custom scanning solution based on a single depth sensor and a fully automatically controlled sensor movement.

The system is based on low cost components (the cost of the full system, including electronic components, platforms, etc. is currently about 300 EUR) and it is modular such that hardware and software components can be easily replaced if new and more powerful ones are available.

First tests show that the current setup should provide sufficient accuracy for the planned applications, however, we plan to improve the software quality and to test the accuracy and usability of the system in real world applications both for scientific studies and industrial applications.

Acknowledgements: This work has been partially supported by the Regione Veneto FSE (Fondo Sociale Europeo) project 1695/1/18/1686/2012: "Ricostruzione 3D e analisi della forma del piede per l'industria calzaturiera"

\section{References}

[1] S. Telfer, et al. The use of 3D surface scanning for the measurement and assessment of the human foot. J Foot Ankle Res, 2010, 5: 19.

[2] B. Sarghie, M. Costea, and D. Liute "Anthropometric Study of the Foot Using 3D Scanning Method and Statistical Analysis." Proceedings of the International Symposium in Knitting and Apparel, Isai, Romania. 2013.

[3] S. Menato et al. Empowering widespread shoe personalization via a 3D foot scanning App. In: Engineering, Technology and Innovation (ICE), 2014 International ICE Conference on. IEEE, 2014. p. 1-7.

[4] Z. Taha et al. A Low Cost 3D Foot Scanner for Custom-Made Sports Shoes. Applied Mechanics and Materials, 2014, 440: 369-372.

[5] S. Izadi et al., KinectFusion: Real-time 3D Reconstruction and Interaction Using a Moving Depth Camera, ACM Symposium on User Interface Software and Technology, October 2011

[6] S. Garrido-Jurado, R. Muñoz-Salinas, FJ Madrid-Cuevas, MJ Marín-Jiménez, Automatic generation and detection of highly reliable fiducial markers under occlusion, Pattern Recognition, Volume 47, Issue 6, June 2014, Pages 2280-2292, ISSN 0031-3203

[7] Processing 2 web site http://www.processing.org/ Last visited on August 27, 2014

[8] Simple OpenNI download site https://code.google.com/p/simple-openni/ Last visited on August 27, 2014

[9] R.B. Rusu, Radu and Steve Cousins. "3d is here: Point cloud library (pcl)." Robotics and Automation (ICRA), 2011 IEEE International Conference on. IEEE, 2011.

[10] CloudCompare (version 2.5.6) [GPL software]. EDF R\&D, Telecom ParisTech. Retrieved from http://www.cloudcompare.org/ on August 27, 2014

[11] C. Herrera, C., J Kannala, and J.Heikkilä. "Joint depth and color camera calibration with distortion correction." Pattern Analysis and Machine Intelligence, IEEE Transactions on 34.10 (2012): 20582064. 